\title{
VEGF: a critical player in neurodegeneration
}

\author{
Erik Storkebaum and Peter Carmeliet \\ Center for Transgene Technology and Gene Therapy, Flanders Interuniversity Institute for Biotechnology, \\ University of Leuven, Leuven, Belgium
}

\begin{abstract}
VEGF is a prototype angiogenic factor, but recent evidence indicates that this growth factor also has direct effects on neural cells. Abnormal regulation of VEGF expression has now been implicated in several neurodegenerative disorders, including motoneuron degeneration. This has stimulated an increasing interest in assessing the therapeutic potential of VEGF as a neuroprotective agent for such neurodegenerative disorders.
\end{abstract}

J. Clin. Invest. 113:14-18 (2004). doi:10.1172/JCI200420682.

No factor is better known for its angiogenic effects than VEGF - this molecule has been implicated in virtually every type of angiogenic disorder, including those associated with cancer, ischemia, and inflammation (1). Recent studies have revealed, however, that VEGF is also involved in neurodegeneration. How can we explain this unexpected finding? It turns out that the role of VEGF in the nervous system is not restricted only to regulating vessel growth: VEGF also has direct effects on different types of neural cells - including even neural stem cells (NSCs). This link between angiogenesis and neurogenesis offers novel opportunities to better decipher the insufficiently understood molecular pathogenesis of many neurodegenerative disorders, and promises to open future avenues for improved treatment.

\section{Vascular effect of VEGF in neurodegeneration}

How can VEGF, and other vascular factors in general, affect neurodegeneration? Recent genetic studies have revealed that reduced VEGF levels cause neurodegeneneration in part by impairing neural tissue perfusion. Indeed, mice with reduced VEGF levels due to a subtle targeted deletion of the hypoxia response element in the promoter of the VEGF gene (VEGF// mice) develop adult-onset motoneuron degeneration (2), reminiscent of amyotrophic lateral sclerosis (ALS). This condition is also known as Lou Gehrig's disease, after the famous baseball player who died of this incurable disorder (3). A follow-up human genetic study documented that "low-VEGF" haplotypes in the VEGF promoter and leader sequence are associated with lower

\footnotetext{
Address correspondence to: Peter Carmeliet, Center for Transgene Technology and Gene Therapy, Flanders Interuniversity Institute for Biotechnology, University of Leuven, Campus Gasthuisberg, Herestraat 49, B-3000 Leuven, Belgium. Phone: 32-16-34-57-74; Fax: 32-16-34-59-90; E-mail: peter.carmeliet@med.kuleuven.ac.be.

Conflict of interest: The authors have declared that no conflict of interest exists.

Nonstandard abbreviations used: neural stem cell (NSC); amyotrophic lateral sclerosis (ALS); endothelial cell (EC).
}

VEGF plasma levels (due to impaired transcription and translation of several VEGF isoforms, including the novel large L-VEGF isoform) and an increased risk of ALS, at least in three European populations (4). As with any other genetic association study, independent highpower replication studies and, even more importantly, additional functional evidence would support the involvement of VEGF in motoneuron degeneration, although the genetic demonstration of a modifier role of VEGF for such a complex disease might be more challenging than is currently anticipated. Reduction of VEGF levels by $50 \%$ is known to significantly impair angiogenesis (5). Somewhat unexpectedly, VEGF $\partial / \partial$ mice, with VEGF levels that are suppressed by only $25 \%$ in the spinal cord, had no major defects in angiogenesis, as measured by capillary density in the spinal cord (2). Yet, neural perfusion was impaired, causing chronic ischemia of motoneurons in the spinal cord (Figure 1) (2). Intriguingly, decreased regional cerebral blood flow has also been reported in patients with $\operatorname{ALS}(6,7)$. These findings, together with the facts that large motoneurons are particularly vulnerable to free radicals generated during ischemia and that release of reactive oxygen species increases with age, suggest that chronic and/or repetitive neural perfusion deficits may set the stage for the development of neuronal damage and, ultimately, neurodegeneration at adult onset. The mechanisms by which low VEGF levels reduce neural perfusion remain to be determined, but they might involve impaired vasoregulation. One possibility is that VEGF affects vascular tone by controlling the release of the vasorelaxant nitric oxide by endothelial cells (ECs). Alternatively, VEGF may be required for the normal functioning of perivascular autonomic nerves, which critically regulate vascular tone and, hence, tissue perfusion. Ongoing studies in VEGF// mice suggest indeed that low VEGF levels cause adult-onset degeneration of these perivascular autonomic nerves (8).

Perfusion deficits have also been documented in other neurodegenerative disorders, including Alzheimer disease and Huntington disease. Evidence 


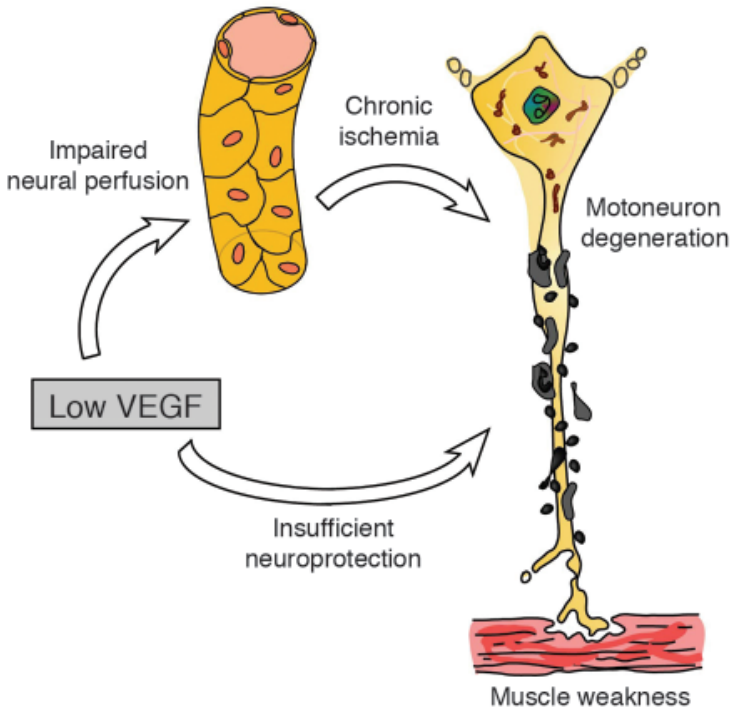

of the presence of perfusion deficits often precedes onset of clinical symptoms, suggesting that they causally contribute to the pathogenesis of these disorders $(9,10)$. Various types of vascular defects have been documented in neurodegenerative disorders (Table 1), including fibrosis and deposition of amorphous substances within the vessel wall and thickening of the basement membrane and interstitial matrix. These abnormalities lead to vessel narrowing, loss of vasoregulation, hypoperfusion, and impaired oxygen diffusion to the neural tissue. Notably, a progressive loss of the microvasculature and reduction of blood supply to the nervous system with increasing age may explain the late onset of many neurodegenerative disorders (11). To what extent VEGF - or other molecules - are involved in the deregulation of neural perfusion in these disorders remains to be elucidated.

\section{Figure 1}

Low VEGF levels cause amyotrophic lateral sclerosis. Low VEGF levels impair spinal cord perfusion and cause chronic ischemia of motoneurons, but also deprive these cells of vital VEGF-dependent survival and neuroprotective signals. Both mechanisms result in adult-onset progressive degeneration of motoneurons, with associated muscle weakness, paralysis, and death - as is typical in amyotrophic lateral sclerosis.

In addition to the above-described etiologic role of VEGF in the initiation phase of neurodegeneration, VEGF may also have additional effects which can occur secondarily in response to the neurodegeneration process itself. These secondary effects likely relate to the fact that VEGF expression is upregulated by ischemic and inflammatory stimuli, which often accompany neurodegeneration. The elevated neural VEGF levels in humans suffering acute focal neural ischemia, diabetes, Alzheimer disease, and vascular dementia may thus be secondary to the ischemia and inflammation in these disorders (12-14), although a primary role cannot be formally excluded to date. Such elevated VEGF levels could initiate a vicious cycle. Indeed, excess VEGF may cause hemangioma formation, microvascular leakage, and fragility. These abnormalities may lead to edema formation and bleeding, and further impair perfusion and tissue oxygenation. The resultant hypoxia may then, in turn, further upregulate VEGF levels again.

\section{Neurotrophic effects of VEGF in neurodegeneration}

There is growing evidence that insufficient neuroprotection by VEGF may be a novel etiologic mechanism of motoneuron degeneration. First, VEGF is expressed in the spinal cord in neurons and glial cells, and is rapidly elevated by hypoxia, whereas VEGFR-2 and neuropilin-1 are expressed in motoneurons (2), indicating that all molecular players required to coordinate a neuropro-

Table 1

Vascular defects in neurodegenerative disorders

Neurodegenerative disease

Amyotrophic lateral sclerosis

Alzheimer disease

Parkinson disease Huntington disease Diabetic neuropathy

CADASIL

Stroke

Prion disease

\section{Vascular defect}

Normal vascular density in the spinal cord but reduced neural perfusion causes chronic ischemia due to low VEGF levels $(2,4)$.

$\beta$-amyloid causes microvascular degeneration (focal swelling, atrophy, and death of vascular cells, disruption of the blood-brain barrier), cerebral amyloid angiopathy (CAA), abnormal vasoregulation, vessel wall rupture, cerebral perfusion deficits, hemorrhages, and infarcts (10). Tortuous thickening of the basement membrane, collagen deposition, CAA, and the presence of plaques/tangles impede diffusion of oxygen. Reduced vascular density correlates with the degree of vascular dementia, but reactive angiogenesis may be induced by cerebral ischemia and upregulation of VEGF (50).

Microvascular basement membrane deposits and capillary dysfunction (51).

Perfusion deficits due to vascular hyporeactivity (9).

Epineural vessels: arteriolar attenuation, venous distension, arteriovenous shunting, new vessel formation. Endoneural vessels: hyalin thickening and increased deposition of amorphous substances in the vessel wall, in association with endothelial cell growth and basement membrane thickening, pericyte loss and - in some cases - capillary pruning and obstruction (52).

Cerebral autosomal dominant arteriopathy with subcortical infarcts and leukoencephalopathy (CADASIL): vascular malformations and degeneration due to smooth muscle cell dysfunction, resulting from Notch3 mutations (53).

Angiogenic cerebral scores correlate with the recovery from stroke (54).

Mobile cells in vessel walls such as dendritic cells and monocytes are implicated in the spread of disease-associated prion protein (55). 
tective effect by VEGF are present in vivo. Second, in $V E G F^{\partial / \partial}$ mice with ALS, insufficient VEGF may deprive motoneurons of critical survival and neuroprotective signals, especially in conditions of hypoxic or excitotoxic stress, and thereby cause motoneuron degeneration (Figure 1) (2). This may explain why VEGF $2 / \partial$ mice are unusually sensitive to transient spinal cord ischemia and remain paralyzed after a minor ischemic insult, while wild-type mice show only a transient clinical deficit (4). Third, neuronal overexpression of VEGFR-2 delays onset of motoneuron degeneration in SOD-1 1 G33 mice - an established mouse model of ALS (8). Fourth, in a mouse model of spinal and bulbar muscular atrophy (also known as SBMA, or Kennedy's disease - a lower motor neuron disease caused by CAG repeat expansion in the androgen receptor gene), reduced spinal cord VEGF protein levels were observed, even in presymptomatic mice (15). Finally, several recent in vitro studies have documented that VEGF protects cultured motoneurons against death in conditions of hypoxia, oxidative stress, and serum deprivation (2), glutamateinduced excitotoxicity $(16,17)$, or mutant SOD-1induced toxicity (18), thus indicating that VEGF has direct neuroprotective effects on motoneurons.

The neuroprotective activity of VEGF might also play a more important (etiologic) role in other neurodegenerative disorders than originally anticipated, although the evidence thus far is largely based on in vitro studies. Various neural cells express one or more of the VEGF receptors (e.g., VEGFR-1, -2 and neuropilin-1) and can thus directly respond to VEGF released by neighboring neural cells $(2,14,19)$. For instance, VEGF stimulates axonal outgrowth in explant cultures of retinal or superior cervical and dorsal root ganglia (14). Furthermore, under conditions of hypoxic, excitotoxic, or oxidative stress, VEGF increases the survival of hippocampal, cortical, cerebellar granule, dopaminergic, autonomic, and sensory neurons $(2,14,16-18,20-23)$. VEGF also stimulates the growth (14) and survival of Schwann cells in hypoxic conditions (24), and increases proliferation and migration of astrocytes $(23,25)$ and microglial cells (26). Future genetic and functional studies in vivo will have to elucidate how important the neuroprotective effect of VEGF is in the pathogenesis of other neurodegenerative disorders.

\section{Neurogenic effects of VEGF}

An impaired regenerative response of neural tissue by adult NSCs has been proposed as a contributory factor in the pathogenesis of neurodegenerative disorders. There is increasing evidence that blood vessels and angiogenic factors such as VEGF play an important role in the control of neurogenesis via crosstalk pathways. For example, ECs and NSCs appear at similar locations and developmental stages in the neural germinal zones (27). In the adult mouse, NSCs are neighbors to ECs and proliferate in small clusters around dividing capillaries ("vascular niches") in the subependymal zone of the lateral ventricle (28). Moreover, when neural progenitors are cocultured with ECs, the maturation, neurite outgrowth, and migration of neurons are enhanced (29). Conditions which increase neural activity and stimulate neurogenesis also trigger angiogenesis $(30$, 31 ), whereas cranial irradiation causes a decrease in both hippocampal neurogenesis and angiogenesis (32). VEGF is produced by ependymal cells at neurogenic sites, stimulates the proliferation of neuronal precursors in cerebral cortical cultures in vitro, and, upon intracerebroventricular infusion, increases neural progenitor growth in the subventricular and subgranular zone of the hippocampal dentate gyrus (33-36), effects that may be mediated via VEGFR-2 (36). VEGF may also promote neurogenesis by stimulating ECs to release neurogenic signals, such as brain-derived neurotrophic factor (37). Notably, intracerebroventricular administration of VEGF reduces infarct size, in part by stimulating neurogenesis and angiogenesis (38). Despite this suggestive evidence, there is currently no formal proof that impaired neurogenesis due to low VEGF levels contributes to neurodegeneration. Another outstanding but challenging question is also whether delivery of VEGF would beneficially affect the outcome of neurodegeneration by stimulating neurogenesis.

\section{VEGF for the treatment of neurodegeneration?}

The emerging evidence for an etiologic role of VEGF in (at least some types of) neurodegeneration provides a rationale for considering the therapeutic potential of VEGF for neurodegenerative disorders, which are mostly incurable. Although the evidence is still circumstantial, VEGF may indeed have such a therapeutic potential. For instance, treatment with VEGF-protected wild-type mice against paralysis induced by short-term spinal cord ischemia (4). VEGF also augmented vascularization and reduced retrograde degeneration of transected corticospinal tract axons, and, remarkably, VEGF stimulated some axons to regenerate across the injured area (39). Intramuscular VEGF gene transfer prevented axonal loss and myelin degeneration and preserved nerve conduction velocities and normal sensory nerve action potentials in a rabbit model of ischemic neuropathy (24) and in patients with critical hind limb ischemia (40). This favorable effect of VEGF was attributed to a vascular effect (i.e., restoration of neural blood flow), as well as to a direct effect on Schwann cells (24). VEGF gene transfer also preserved neural flow by preventing regression of the vasa nervorum, resulting in restoration of peripheral nerve function in rats with streptozotocin-induced diabetes (41). Furthermore, VEGF improved nerve regeneration by stimulating the outgrowth of Schwann cells and blood vessels (42). In a rat model of Parkinson disease, continuous local production of VEGF improved amphetamine-induced rotational behavior (43). VEGF can also have favorable effects on the recovery of an ischemic brain insult. Administration of VEGF via intravenous or intracerebrovascular infusion, or via topical application on the cortex, enhanced angiogenesis in the 
penumbra and improved neural recovery (44), reduced edema formation and infarct volume $(45,46)$, and amplified neurogenesis (38), while knockdown of VEGF after ischemic stroke enlarged the infarct volume (47). Some caution, however, is warranted, as other studies suggest that delivery of a VEGF trap reduces edema formation and spares ischemic brain tissue (48), while infusion of VEGF induces vascular leakage, with resultant hemorrhagic transformation of the ischemic lesions (44). VEGF has also been documented to have beneficial effects in epilepsy, as infusion of VEGF into rat brains protected CA1 and CA3 hippocampal regions against neuronal cell damage induced by epilepsy (49). Overall, although additional work is required, these studies highlight the important biological role of VEGF in neurodegenerative disorders and provide sufficient rationale for evaluating the potential therapeutic utility of VEGF in their management.

\section{Acknowledgments}

The authors wish to thank Ann Vandenhoeck for the artwork, and all previous and current collaborators for assistance in this study.

1. Carmeliet, P. 2003. Angiogenesis in health and disease. Nat. Med. 9:653-660.

2. Oosthuyse, B., et al. 2001. Deletion of the hypoxia-response element in the vascular endothelial growth factor promoter causes motor neuron degeneration. Nat. Genet. 28:131-138.

3. Cleveland, D.W., and Rothstein, J.D. 2001. From Charcot to Lou Gehrig: deciphering selective motor neuron death in ALS. Nat. Rev. Neurosci. 2:806-819.

4. Lambrechts, D., et al. 2003. VEGF is a modifier of amyotrophic lateral sclerosis in mice and humans and protects motoneurons against ischemic death. Nat. Genet. 34:383-394.

5. Carmeliet, P., et al. 1996. Abnormal blood vessel development and lethality in embryos lacking a single VEGF allele. Nature. 380:435-439.

6. Kobari, M., Obara, K., Watanabe, S., Dembo, T., and Fukuuchi, Y. 1996. Local cerebral blood flow in motor neuron disease: correlation with clinical findings. J. Neurol. Sci. 144:64-69.

7. Waldemar, G., Vorstrup, S., Jensen, T.S., Johnsen, A., and Boysen, G. 1992. Focal reductions of cerebral blood flow in amyotrophic lateral sclerosis: a [99mTc]-d,l-HMPAO SPECT study. J. Neurol. Sci. 107:19-28.

8. Storkebaum, E., et al. 2003. VEGF, a modifier of motor neuron degeneration in SOD1 G93A mice, protects against motor neuron loss after spinal cord ischemia: evidence for a vascular hypothesis. Program No. 602.11. 2003. Abstract Viewer/Itinerary Planner. Society for Neuroscience. Washington, DC, USA. http://sfn.scholarone.com.

9. Deckel, A.W., and Duffy, J.D. 2000. Vasomotor hyporeactivity in the anterior cerebral artery during motor activation in Huntington's disease patients. Brain Res. 872:258-261.

10. Kalaria, R.N. 2002. Small vessel disease and Alzheimer's dementia: pathological considerations. Cerebrovasc. Dis. 13:48-52.

11. Buijs, P.C., et al. 1998. Effect of age on cerebral blood flow: measurement with ungated two-dimensional phase-contrast MR angiography in 250 adults. Radiology. 209:667-674.

12. Tarkowski, E., et al. 2002. Increased intrathecal levels of the angiogenic factors VEGF and TGF-beta in Alzheimer's disease and vascular dementia. Neurobiol. Aging. 23:237-243.

13. Samii, A., Unger, J., and Lange, W. 1999. Vascular endothelial growth factor expression in peripheral nerves and dorsal root ganglia in diabetic neuropathy in rats. Neurosci. Lett. 262:159-162.

14. Sondell, M., Lundborg, G., and Kanje, M. 1999. Vascular endothelial growth factor has neurotrophic activity and stimulates axonal outgrowth, enhancing cell survival and Schwann cell proliferation in the peripheral nervous system. J. Neurosci. 19:5731-5740.

15. Thomas, P.S., et al. 2003. SBMA motor neuronopathy in AR YAC CAG100 transgenic mice may involve altered expression of VEGF. Program No. 413.2. 2003. Abstract Viewer/Itinerary Planner. Society for Neuroscience. Washington, DC, USA. http://sfn.scholarone.com.

16. Matsuzaki, H., et al. 2001. Vascular endothelial growth factor rescues hippocampal neurons from glutamate-induced toxicity: signal trans- duction cascades. FASEB J. 15:1218-1220.

17. Svensson, B., et al. 2002. Vascular endothelial growth factor protects cultured rat hippocampal neurons against hypoxic injury via an antiexcitotoxic, caspase-independent mechanism. J. Cereb. Blood Flow Metab. 22:1170-1175.

18. Li, B., Xu, W., Luo, C., Gozal, D., and Liu, R. 2003. VEGF-induced activation of the PI3-K/Akt pathway reduces mutant SOD1-mediated motor neuron cell death. Brain Res. Mol. Brain Res. 111:155-164.

19. Sondell, M., and Kanje, M. 2001. Postnatal expression of VEGF and its receptor flk-1 in peripheral ganglia. Neuroreport. 12:105-108.

20. Jin, K.L., Mao, X.O., and Greenberg, D.A. 2000. Vascular endothelial growth factor: direct neuroprotective effect in in vitro ischemia. Proc. Natl. Acad. Sci. U. S. A. 97:10242-10247.

21. Jin, K.L., Mao, X.O., and Greenberg, D.A. 2000. Vascular endothelial growth factor rescues HN33 neural cells from death induced by serum withdrawal. J. Mol. Neurosci. 14:197-203.

22. Ogunshola, O.O., et al. 2002. Paracrine and autocrine functions of neuronal vascular endothelial growth factor (VEGF) in the central nervous system. J. Biol. Chem. 277:11410-11415.

23. Silverman, W.F., Krum, J.M., Mani, N., and Rosenstein, J.M. 1999. Vascular, glial and neuronal effects of vascular endothelial growth factor in mesencephalic explant cultures. Neuroscience. 90:1529-1541.

24. Schratzberger, P., et al. 2000. Favorable effect of VEGF gene transfer on ischemic peripheral neuropathy. Nat. Med. 6:405-413.

25. Krum, J.M., Mani, N., and Rosenstein, J.M. 2002. Angiogenic and astroglial responses to vascular endothelial growth factor administration in adult rat brain. Neuroscience. 110:589-604.

26. Forstreuter, F., Lucius, R., and Mentlein, R. 2002. Vascular endothelial growth factor induces chemotaxis and proliferation of microglial cells. J. Neuroimmunol. 132:93-98.

27. Zerlin, M., and Goldman, J.E. 1997. Interactions between glial progenitors and blood vessels during early postnatal corticogenesis: blood vessel contact represents an early stage of astrocyte differentiation. J. Comp. Neurol. 387:537-546.

28. Palmer, T.D., Willhoite, A.R., and Gage, F.H. 2000. Vascular niche for adult hippocampal neurogenesis. J. Comp. Neurol. 425:479-494.

29. Leventhal, C., Rafii, S., Rafii, D., Shahar, A., and Goldman, S.A. 1999. Endothelial trophic support of neuronal production and recruitment from the adult mammalian subependyma. Mol. Cell. Neurosci. 13:450-464.

30. Black, J.E., Isaacs, K.R., Anderson, B.J., Alcantara, A.A., and Greenough, W.T. 1990. Learning causes synaptogenesis, whereas motor activity causes angiogenesis, in cerebellar cortex of adult rats. Proc. Natl. Acad. Sci. U. S. A. 87:5568-5572.

31. Kokaia, Z., and Lindvall, O. 2003. Neurogenesis after ischaemic brain insults. Curr. Opin. Neurobiol. 13:127-132.

32. Monje, M.L., Mizumatsu, S., Fike, J.R., and Palmer, T.D. 2002. Irradiation induces neural precursor-cell dysfunction. Nat. Med. 8:955-962.

33. Yang, K., and Cepko, C.L. 1996. Flk-1, a receptor for vascular endothelial growth factor (VEGF), is expressed by retinal progenitor cells. J. Neurosci. 16:6089-6099.

34. Yourey, P.A., Gohari, S., Su, J.L., and Alderson, R.F. 2000. Vascular endothelial cell growth factors promote the in vitro development of rat photoreceptor cells. J. Neurosci. 20:6781-6788.

35. Jin, K., et al. 2002. Vascular endothelial growth factor (VEGF) stimulates neurogenesis in vitro and in vivo. Proc. Natl. Acad. Sci. U. S. A. 99:11946-11950.

36. Zhu, Y., Jin, K., Mao, X.O., and Greenberg, D.A. 2003. Vascular endothelial growth factor promotes proliferation of cortical neuron precursors by regulating E2F expression. FASEB J. 17:186-193.

37. Louissaint, A., Jr., Rao, S., Leventhal, C., and Goldman, S.A. 2002. Coordinated interaction of neurogenesis and angiogenesis in the adult songbird brain. Neuron. 34:945-960.

38. Sun, Y., et al. 2003. VEGF-induced neuroprotection, neurogenesis, and angiogenesis after focal cerebral ischemia. J. Clin. Invest. 111:1843-1851. doi:10.1172/JCI200317977.

39. Facchiano, F., et al. 2002. Promotion of regeneration of corticospinal tract axons in rats with recombinant vascular endothelial growth factor alone and combined with adenovirus coding for this factor. J. Neurosurg. 97:161-168.

40. Simovic, D., Isner, J.M., Ropper, A.H., Pieczek, A., and Weinberg, D.H. 2001. Improvement in chronic ischemic neuropathy after intramuscular phVEGF165 gene transfer in patients with critical limb ischemia. Arch. Neurol. 58:761-768.

41. Schratzberger, P., et al. 2001. Reversal of experimental diabetic neuropathy by VEGF gene transfer. J. Clin. Invest. 107:1083-1092.

42. Sondell, M., Lundborg, G., and Kanje, M. 1999. Vascular endothelial growth factor stimulates Schwann cell invasion and neovascularization of acellular nerve grafts. Brain Res. 846:219-228.

43. Yasuhara, T., et al. 2003. Vascular endothelial growth factor has a neuroprotective effect on Parkinson's disease. Program No. 233.6. 2003. Abstract Viewer/Itinerary Planner. Society for Neuroscience. Washington, DC, USA. http://sfn.scholarone.com.

44. Zhang, Z.G., et al. 2000. VEGF enhances angiogenesis and promotes bloodbrain barrier leakage in the ischemic brain. J. Clin. Invest. 106:829-838. 
45. Harrigan, M.R., Ennis, S.R., Sullivan, S.E., and Keep, R.F. 2003. Effects of intraventricular infusion of vascular endothelial growth factor on cerebral blood flow, edema, and infarct volume. Acta Neurochir. (Wien). 145:49-53.

46. Hayashi, T., Abe, K., and Itoyama, Y. 1998. Reduction of ischemic damage by application of vascular endothelial growth factor in rat brain after transient ischemia. J. Cereb. Blood Flow Metab. 18:887-895.

47. Yang, Z.J., et al. 2002. Role of vascular endothelial growth factor in neuronal DNA damage and repair in rat brain following a transient cerebral ischemia. J. Neurosci. Res. 70:140-149.

48. van Bruggen, N., et al. 1999. VEGF antagonism reduces edema formation and tissue damage after ischemia/reperfusion injury in the mouse brain. J. Clin. Invest. 104:1613-1620.

49. Croll, S.D., Goodman, J.H., and Scharfman, H.E. 2003. Vascular endothelial growth factor (VEGF) in seizures: a double-edged sword. In Recent advances in epilepsy research. D.K. Binder and H.E. Scharfman, editors. Kluwer Academic/Plenum Publishers. New York, New York, USA. In press.
50. Vagnucci, A.H., Jr., and Li, W.W. 2003. Alzheimer's disease and angiogenesis. Lancet. 361:605-608.

51. Farkas, E., De Jong, G.I., de Vos, R.A., Jansen Steur, E.N., and Luiten, P.G. 2000. Pathological features of cerebral cortical capillaries are doubled in Alzheimer's disease and Parkinson's disease. Acta Neuropathol. (Berl) 100:395-402.

52. Boulton, A.J., and Malik, R.A. 1998. Diabetic neuropathy. Med. Clin. North Am. 82:909-929.

53. Kalimo, H., Ruchoux, M.M., Viitanen, M., and Kalaria, R.N. 2002. CADASIL: a common form of hereditary arteriopathy causing brain infarcts and dementia. Brain Pathol. 12:371-384.

54. Krupinski, J., Kaluza, J., Kumar, P., Kumar, S., and Wang, J.M. 1994. Role of angiogenesis in patients with cerebral ischemic stroke. Stroke. 25:1794-1798.

55. Koperek, O., et al. 2002. Disease-associated prion protein in vessel walls. Am. J. Pathol. 161:1979-1984. 